\title{
When can antiepileptic drugs be safely withdrawn?
}

\section{Y Hart}

\section{A further attempt to assess risk of recurrent seizures if medication is discontinued}

O ne of the more difficult questions in the management of people with epilepsy is, "When can antiepileptic drugs be safely withdrawn? For adults, the reasons for continuing medication are often as much social as medical, and the issue of driving is frequently a major factor in the equation. Other considerations include the impact of recurrent seizures on employment, the possibility of teratogenicity in women continuing medication, the risk of injury secondary to seizures, and, increasingly recognised in recent years, the risk of sudden unexpected death in epilepsy (SUDEP). The situation is complicated by the fact that not all patients experiencing a recurrence of their seizures regain seizure control promptly when antiepileptic drugs are restarted.

The study by Specchio et al (this issue, pp 22-25) $)^{2}$ is a further attempt to assist the clinician in advising the patient on the probable risk of recurrent seizures if they do discontinue medication. As in the earlier Medical Research Council Antiepileptic Drug Withdrawal Study (1991), ${ }^{3}$ to take part patients had to have been seizure free for at least 2 years, and to consent to drug withdrawal, thus introducing a possible source of selection bias. The current study was carried out from a tertiary centre (suggesting that the patients might have relatively severe epilepsy), but included only those who had been taking monotherapy for the previous year (possibly indicating that, on the contrary, their epilepsy was "mild"). Nevertheless, the results are remarkably similar to those from the MRC study, ${ }^{3}$ with the probability of remaining seizure free at 2 years being $82 \%$ in those continuing treatment and $57 \%$ in those withdrawing from antiepileptic drugs (compared with $78 \%$ and $59 \%$ in the MRC study). As in the MRC study, duration of active disease and duration of remission both influenced the likelihood of recurrence.

These figures are helpful in corroborating the results of the MRC study, and will certainly inform us in counselling patients who wish to stop medication. It seems likely that the high rate of seizure recurrence will deter many patients (particularly those with driving licences) from withdrawing medication for considerably longer than the 2 years of seizure freedom which is currently commonly recommended. However, what is most important for many people with epilepsy, who have the same difficulty as the rest of us in conceptualising risks, is whether they, individually, will have further seizures if they discontinue their antiepileptic drugs. At present, except in the case of such specific epileptic syndromes as juvenile myoclonic epilepsy and benign epilepsy of childhood with centrotemporal spikes, we remain unable to answer this question. Various attempts have been made at quantifying the effect of such factors as the EEG, period of freedom from seizures, and age at onset of epilepsy, ${ }^{4}$ and they may improve our predictions to some extent, but it seems likely that any advances will be limited until we succeed in breaking down the hotchpotch of conditions we call "epilepsy" into its constituent syndromes more accurately.

J Neurol Neurosurg Psychiatry 2002;72:5-5

\section{Author's affiliations}

Y Hart, Department of Neurology, Radcliffe Infirmary, Woodstock Road, Oxford OX2 6HE, UK; yvonnehart@compuserve.com

\section{REFERENCES}

1 Todt $\mathbf{H}$. The late prognosis of epilepsy in childhood: results of a prospective follow-up study. Epilepsia 1984;25:137-44.

2 Specchio LM, Tramacere, Neve A La, et al. Discontinuing antiepileptic drugs in patients who are seizure-free on monotherapy. $J$ Neurol Neurosurg Psychiatry 2002;72:22-5.

3 Medical Research Council Antiepileptic Drug Withdrawal Study Group. A randomized study of antiepileptic drug withdrawal in patients in remission of epilepsy. Lancet 1991;337:1175-80.

4 Berg AT, Shinnar S, Relapse following discontinuation of antiepileptic drugs: meta-analysis. Neurology 1994;44:601-8. 